\title{
Stressed? Time to vesiculate...
}

DOI:

10.1038/nrmicro1608

\section{URLs}

Escherichia coli

http://www.ncbi.nlm.nih.gov/ entrez/query.fcgi?db=genome $\operatorname{prj} \& \mathrm{cmd}=$ RetrieveEdopt $=$ Over viewElist_uids $=12319$

Salmonella enterica serovar Typhimurium

http://www.ncbi.nlm.nih.gov/ entrez/query.fcgi?db=genome prj\&cmd=Retrieve\&dopt=Ove view\&list_uids $=12320$
Gram-negative bacteria bud off outer membrane-derived vesicles that contain periplasmic components throughout growth. Although these vesicles can mediate virulence protein export, a more general physiological role for vesicle production has remained elusive. Now, McBroom and Kuehn show that vesiculation is a regulated response to envelope stress.

Stressors such as oxidants, metals and heat can damage bacterial cells, and stress leads to the toxic accumulation of misfolded proteins in the cell envelope. In Escherichia coli (and other Gram-negative bacteria) several complementary extracytoplasmic pathways are activated in response to envelope stress to protect cells. These include the $\sigma^{\mathrm{E}}$ pathway (responds to misfolded outer-membrane proteins), the Cpx pathway (monitors cell-surface-protein assembly), the Psp system (senses dissipation of the proton motive force) and the Bae pathway (responds to antimicrobial compounds). These survival pathways are also considered to be crucial to bacterial pathogens because pathogens are subjected to envelope stress during the infection process.

Initially, McBroom and Kuehn noticed that disrupting the $\sigma^{\mathrm{E}}$ envelope stress pathway by knocking out key signalling proteins resulted in a massive increase in vesiculation in both E. coli and Salmonella enterica serovar Typhimurium. But after carefully examining the levels of $\sigma^{\mathrm{E}}$ in their collection of $E$. coli vesiculation mutants, they failed to find a clear link between the activity of this alternate $\sigma$ factor and vesiculation. Instead, examination of $\sigma^{\mathrm{E}}$ mutants and wildtype cells showed that vesiculation increased in response to envelope damage. Vesiculation was not regulated by the $c p x, p s p$ or bae pathways either, so vesiculation is a new and independent envelope stress response.

The authors reasoned that vesiculation might be protective, and indeed, increased vesiculation improved the survival rates of bacteria that were exposed to envelope stressors, including the production of a periplasmic, misfolded and toxic LamB protein. Analysing vesicle cargoes revealed that although the response to some stressors led to the non-selective bulk export of periplasmic contents, other stressors are selectively removed.

This novel stress response provides bacteria with a means to rid cells of toxic and misfolded proteins. The next step will be to understand the underlying mechanism(s) of vesiculation.

Susan Jones

ORIGINAL RESEARCH PAPER McBroom, A. J. Kuehn, M. J. Release of outer membrane vesicles by Gram-negative bacteria is a novel envelope stress response Mol. Microbiol. 63, 545-558 (2007)

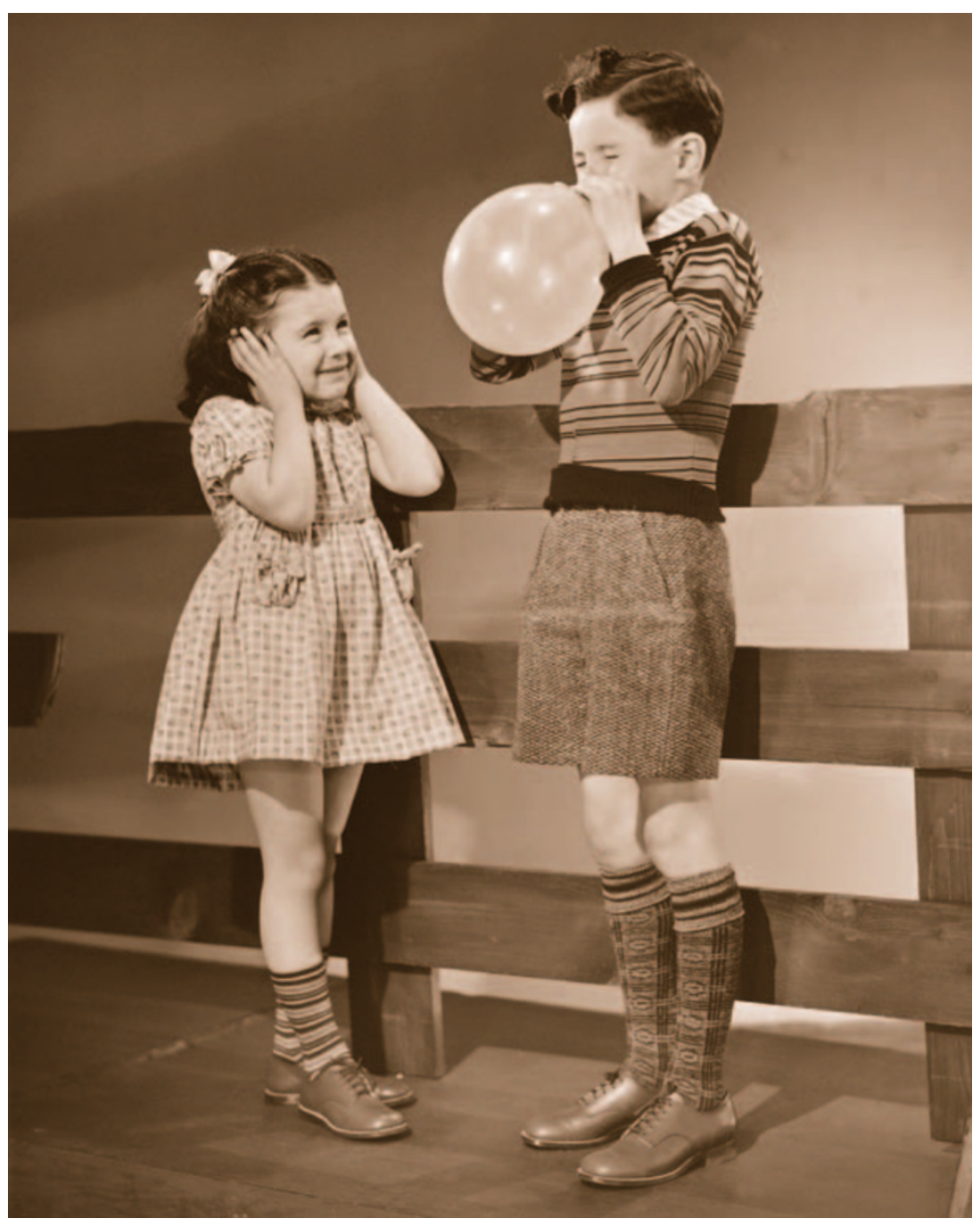

